International Mathematical Forum, 2, 2007, no. 60, 2963 - 2970

\title{
Some Limit Theorems on Randomly Weighted Sums for Inhomogeneous Markov Chains ${ }^{1}$
}

\author{
Zhong-zhi Wang \\ Faculty of Mathematics \& Physics \\ AnHui University of Technology \\ Ma'anshan, 243002, China \\ wzz30@ahut.edu.cn \\ Fang-qing Ding \\ School of Mathematics \& Physics \\ HeFei University, HeFei, 230601, China
}

\begin{abstract}
Some convergence theorems are established for randomly weighted sums of the form $\Gamma_{n}=\sum_{k=1}^{n} w_{k} g_{k}\left(\xi_{k-1}, \xi_{k}\right)$ where $\left\{\xi_{n}, n \geq 0\right\}$ is an inhomogeneous Markov chains. As a special case, some limit theorems on random selection for inhomogeneous Markov chains are also obtained
\end{abstract}

Mathematics Subject Classification: 60F15; 60J10

Keywords: inhomogeneous Markov chains; randomly weighted sums; martingale; random selection; strong limit theorem

\section{Introduction}

Let $(\Omega, \mathcal{F}, P)$ be a probability space with a distinguished nondecreasing family $\left\{\mathcal{F}_{n}, n \geq 1\right\}$ of $\sigma$-algebras $\mathcal{F}_{0} \subseteq \mathcal{F}_{1} \subseteq \cdots \subseteq \mathcal{F}$.

Let $\left\{\xi_{n}, n \geq 0\right\}$ be an inhomogeneous Markov chain taking values in $\mathcal{E}=$ $\left\{t_{1}, t_{2}, \cdots, t_{m}\right\}$ with initial distribution (1.1) and transition matrices (1.2)

$$
\begin{gathered}
\left(q\left(t_{1}\right), q\left(t_{2}\right), \cdots, q\left(t_{m}\right)\right) \\
P_{n}=\left(p_{n}\left(t_{i}, t_{j}\right)\right)_{m \times m}, t_{i}, t_{j} \in \mathcal{E}, n=1,2, \cdots
\end{gathered}
$$

\footnotetext{
${ }^{1}$ Supported by Science Foundation of the Education Committee of AnHui Province (2006Kj 246B) and HeFei University research grant: 606040.
} 
where $p_{n}\left(t_{i}, t_{j}\right)=P\left(\xi_{n}=t_{j} \mid \xi_{n-1}=t_{i}\right)$, then

$$
P\left(\xi_{0}=x_{0}, \cdots, \xi_{n}=x_{n}\right)=q\left(x_{0}\right) \prod_{k=1}^{n} p_{k}\left(x_{k-1}, x_{k}\right), n>1
$$

when considering the randomly weighted sums on functional of inhomogeneous Markov chain, let

$$
f_{1}\left(x_{0}\right), f_{2}\left(x_{0}, x_{1}\right), \cdots, f_{n}\left(x_{0}, \cdots x_{n-1}\right), \cdots
$$

be a series of measurable functions.

Define

$$
w_{n}=f_{n}\left(\xi_{0}, \cdots \xi_{n-1}\right), n=1,2, \cdots
$$

which $\left\{w_{n}, n \geq 1\right\}$ is a predictable sequence (see e.g. [1],[2]).

Definition 1. Let $\left\{\xi_{n}, n \geq 0\right\},\left\{w_{n}, n \geq 1\right\}$ be defined as before, $g_{n}(\cdot, \cdot),(n \geq$ $1)$ be any functions of two variables defined in $\mathcal{E}^{2}$. The random variables

$$
\Gamma_{n}=\sum_{k=1}^{n} w_{k} g_{k}\left(\xi_{k-1}, \xi_{k}\right)
$$

is said to be the randomly weighted sums of $\left\{g_{n}\left(\xi_{n-1}, \xi_{n}\right), n \geq 1\right\}$ respect to $\left\{w_{n}, n \geq 1\right\}$.

Lemma 1. (see e.g. [2])Let $\left\{\xi_{n}, \mathcal{F}_{n}, n \geq 1\right\}$ be a martingale difference sequence. Then

$$
\sum_{n=1}^{\infty} E\left[\xi_{n}^{2} \mid \mathcal{F}_{n-1}\right]<\infty \text { implies } \sum_{n} \xi_{n} \text { converges }
$$

\section{Main results and proofs}

Theorem 1. Let $\left\{\xi_{n}, n \geq 0\right\}$ be an inhomogeneous Markov chain with the initial distribution of (1.1) and the transition matrices of (1.2), $g_{n}(\cdot, \cdot),(n \geq 1)$, $\left\{w_{n}, n \geq 1\right\}$ be defined as before, $\left\{a_{n}, n \geq 1\right\}$ is a nondecreasing predictable sequence with $a_{n} \uparrow \infty$ a.s. If

$$
\sup _{k \geq 1}\left|w_{k}\right|<\infty \quad \text { a.s. }
$$

and

$$
\sum_{n=1}^{\infty} a_{n}^{-2} E\left[g_{n}^{2}\left(\xi_{n-1}, \xi_{n}\right)\right]<+\infty
$$

then

$$
\lim _{n} a_{n}^{-1} \sum_{k=1}^{n} w_{k}\left\{g_{k}\left(\xi_{k-1}, \xi_{k}\right)-E\left[g_{k}\left(\xi_{k-1}, \xi_{k}\right) \mid \xi_{k-r}\right]\right\}=0 \quad \text { a.s. }
$$

for all $r \geq 1$ (where $\xi_{-n}=$ const., $n \geq 1$ ) 
Proof: Let

$$
\begin{gathered}
\eta_{k}^{(1)}=g_{k}\left(\xi_{k-1}, \xi_{k}\right)-E\left[g_{k}\left(\xi_{k-1}, \xi_{k}\right) \mid \xi_{k-1}\right], \quad k \geq 1 \\
\eta_{k}^{(l)}=E\left[g_{k}\left(\xi_{k-1}, \xi_{k}\right) \mid \xi_{k-l+1}\right]-E\left[g_{k}\left(\xi_{k-1}, \xi_{k}\right) \mid \xi_{k-l}\right], \quad k \geq 2, \quad l \geq 2
\end{gathered}
$$

It is easy to see that, for every $l,\left\{\eta_{k}^{(l)}, k \geq 1\right\}$ is a martingale difference sequence. In fact, from the properties of conditional expectation and Markovin, we have

$$
\begin{aligned}
E\left[\eta_{k}^{(l)} \mid \eta_{1}^{(l)}, \cdots, \eta_{k-1}^{(l)}\right] & =E\left\{E\left[\eta_{k}^{(l)} \mid \xi_{0}, \cdots, \xi_{k-l}\right] \mid \eta_{1}^{(l)}, \cdots, \eta_{k-1}^{(l)}\right\} \\
& =E\left\{E\left[\eta_{k}^{(l)} \mid \xi_{k-l}\right] \mid \eta_{1}^{(l)}, \cdots, \eta_{k-1}^{(l)}\right\}
\end{aligned}
$$

and

$$
\begin{aligned}
& E\left\{E\left[g_{k}\left(\xi_{k-1}, \xi_{k}\right) \mid \xi_{k-l+1}\right] \mid \xi_{k-l}\right\} \\
= & E\left\{E\left[g_{k}\left(\xi_{k-1}, \xi_{k}\right) \mid \xi_{0}, \cdots, \xi_{k-l+1}\right] \mid \xi_{k-l}\right\} \\
= & E\left\{E\left[g_{k}\left(\xi_{k-1}, \xi_{k}\right) \mid \xi_{k-l}\right] \mid \xi_{0}, \cdots, \xi_{k-l+1}\right\} \\
= & E\left[g_{k}\left(\xi_{k-1}, \xi_{k}\right) \mid \xi_{k-l}\right]
\end{aligned}
$$

Hence $E\left[\eta_{k}^{(l)} \mid \xi_{k-l}\right]=0$. This together with (2.6) we have

$$
E\left[\eta_{k}^{(l)} \mid \eta_{1}^{(l)}, \cdots, \eta_{k-1}^{(l)}\right]=0
$$

i.e. $\left\{\eta_{n}^{(l)}, \mathcal{F}_{n}, n \geq 1\right\}$ is a martingale difference sequence.

From the inequality of Jensen for conditional expectation, we have

$$
E\left\{E\left[g_{k}\left(\xi_{k-1}, \xi_{k}\right) \mid \xi_{k-l}\right]\right\}^{2} \leq E\left\{E\left[g_{k}^{2}\left(\xi_{k-1}, \xi_{k}\right) \mid \xi_{k-l}\right]\right\}=E\left[g_{k}^{2}\left(\xi_{k-1}, \xi_{k}\right)\right]
$$

and

$$
\left\{g_{k}\left(\xi_{k-1}, \xi_{k}\right)-E\left[g_{k}\left(\xi_{k-1}, \xi_{k}\right) \mid \xi_{k-l}\right]\right\}^{2} \leq 2\left\{g_{k}^{2}\left(\xi_{k-1}, \xi_{k}\right)+E\left[g_{k}^{2}\left(\xi_{k-1}, \xi_{k}\right) \mid \xi_{k-l}\right]\right\}
$$

Therefore

$$
E\left[\left(\eta_{n}^{(l)}\right)^{2} \mid \mathcal{F}_{n-1}\right] \leq 4 E\left[g_{n}^{2}\left(\xi_{n-1}, \xi_{n}\right)\right]
$$

Fixed a constant $L>0$, by (2.2) and (2.8), we have

$$
E\left[\left(\frac{w_{n} I_{\left[w_{n} \leq L\right]}\left[\eta_{n}^{(l)}\right.}{a_{n}}\right)^{2} \mid \mathcal{F}_{n-1}\right] \leq \frac{L^{2}}{a_{n}^{2}} E\left[\left(\eta_{n}^{(l)}\right)^{2} \mid \mathcal{F}_{n-1}\right] \leq \frac{4 L^{2}}{a_{n}^{2}} E\left[g_{n}^{2}\left(\xi_{n-1}, \xi_{n}\right)\right]
$$

This and the condition (2.1) yield

$$
\lim _{n} a_{n}^{-1} \sum_{k=1}^{n} w_{k} \eta_{k}^{(l)}=0
$$

Note that

$$
g_{k}\left(\xi_{k-1}, \xi_{k}\right)-E\left[g_{k}\left(\xi_{k-1}, \xi_{k}\right) \mid \xi_{k-r}\right]=\eta_{k}^{(1)}+\eta_{k}^{(2)}+\cdots+\eta_{k}^{(r)}
$$

The theorem 1 follows immediately. 


\section{Random Selection and Some Limit Theorems}

The concept of random selection was come from the system of gambling, The importance of this problem was first recognized by von Mises, who introduced it as a fundamental axiom (see.[3]). The relationship between this topic and the logical fundamental of probability is still be paid close attentions by many scholars(see e.g.[4],[5]). The purpose of this paper is to extend some results of random selection to inhomogeneous Markov chain by means of the notion of transform.

In the following paper, we always assume $\left\{w_{n}, n \geq 1\right\}$ be random selection functions, i.e. $w_{n}\left(x_{0}, \cdots x_{n-1}\right)$ is a Bool function taking values in $\{0,1\}$, When consider the problem of random selection,choose subsequences of

$$
\xi_{0}, \xi_{1}, \cdots, \xi_{n}
$$

and

$$
\left(\xi_{0}, \xi_{1}\right),\left(\xi_{1}, \xi_{2}\right) \cdots,\left(\xi_{n-1}, \xi_{n}\right)
$$

according to $w_{k}$. Whether $\xi_{k}$ or $\left(\xi_{k-1}, \xi_{k}\right)$ is chosen depends on whether $w_{k}$ takes 1 or not, denote

$$
\begin{gathered}
\sigma_{n}=\sum_{k=1}^{n} w_{k}, \\
\tau_{n}\left(t_{j} ; \omega\right)=\sum_{k=1}^{n} w_{k} \delta_{t_{j}}\left(\xi_{k}\right), \\
\tau_{n}\left(t_{i}, t_{j} ; \omega\right)=\sum_{k=1}^{n} w_{k} \delta_{t_{i}}\left(\xi_{k-1}\right) \delta_{t_{j}}\left(\xi_{k}\right)
\end{gathered}
$$

Note that

$$
E\left[g_{k}\left(\xi_{k-1}, \xi_{k}\right) \mid \xi_{k-1}\right]=\sum_{t_{j} \in \mathcal{E}} g_{k}\left(\xi_{k-1}, t_{j}\right) p_{k}\left(\xi_{k-1}, t_{j}\right)
$$

Let $\delta_{t_{j}}(\cdot)$ be Kronecker $\delta$ function. We can get some corollaries from theorem 1.

Theorem 2. Let $\left\{\xi_{n}, n \geq 0\right\}$ be an inhomogeneous Markov chain, $g(\cdot, \cdot)$ be a function of two variables defined in $\mathcal{E}^{2},\left\{w_{n}, n \geq 1\right\}, \sigma_{n}$ defined as before with $\sigma_{n} \uparrow \infty$ a.s., then

$$
\lim _{n} \sigma_{n}^{-1} \sum_{k=1}^{n} w_{k}\left[g\left(\xi_{k-1}, \xi_{k}\right)-\sum_{t_{j} \in \mathcal{E}} g\left(\xi_{k-1}, t_{j}\right) p_{k}\left(\xi_{k-1}, t_{j}\right)\right]=0 \quad \text { a.s. }
$$

Proof: We calculate

$$
E\left[g^{2}\left(\xi_{k-1}, \xi_{k}\right) \mid \xi_{k-1}\right]=\sum_{t_{j} \in \mathcal{E}} g^{2}\left(\xi_{k-1}, t_{j}\right) p_{k}\left(\xi_{k-1}, t_{j}\right) \leq \max _{t_{i}, t_{j}} g^{2}\left(t_{i}, t_{j}\right), k \geq 1
$$


Since

$$
\begin{aligned}
& \sum_{k=1}^{n} \sigma_{k}^{-2} w_{k}^{2} E\left[g^{2}\left(\xi_{k-1}, \xi_{k}\right) \mid \xi_{k-1}\right] \\
\leq & \max _{t_{i}, t_{j}} g^{2}\left(t_{i}, t_{j}\right) \sum_{k=1}^{\infty}\left(\frac{w_{k}}{\sigma_{k}}\right)^{2}=\max _{t_{i}, t_{j}} g^{2}\left(t_{i}, t_{j}\right) \sum_{k=1}^{\infty} \frac{1}{k^{2}}<\infty
\end{aligned}
$$

Therefore (3.2) follows immediately from Theorem 1.

Corollary 1. Let $\left\{\xi_{n}, n \geq 0\right\}$ be an inhomogeneous Markov chain and $g(x, y)$, $\left\{w_{n}, n \geq 1\right\}, \tau_{n}\left(t_{j} ; \omega\right), \tau_{n}\left(t_{i}, t_{j} ; \omega\right), \sigma_{n}$ be defined as before, then

$$
\lim _{n} \sigma_{n}^{-1}\left[\tau_{n}\left(t_{j} ; \omega\right)-\sum_{k=1}^{n} w_{k} p_{k}\left(\xi_{k-1}, t_{j}\right)\right]=0 \quad \text { a.s. }
$$

Proof: Let $g(x, y)=\delta_{t_{j}}(y), x, y \in \mathcal{E}$ in Theorem 2, we have

$$
\begin{aligned}
& \sum_{k=1}^{n} w_{k}\left\{g\left(\xi_{k-1}, \xi_{k}\right)-\sum_{t_{j} \in \mathcal{E}} g\left(\xi_{k-1}, t_{j}\right) p_{k}\left(\xi_{k-1}, t_{j}\right)\right\} \\
= & \sum_{k=1}^{n} w_{k}\left\{\delta_{t_{j}}\left(\xi_{k}\right)-\sum_{y \in \mathcal{E}} \delta_{t_{j}}(y) p_{k}\left(\xi_{k-1}, x\right)\right\} \\
= & \left.\tau_{n}\left(t_{j} ; \omega\right)-\sum_{k=1}^{n} w_{k} p_{k}\left(\xi_{k-1}, t_{j}\right)\right]
\end{aligned}
$$

The corollary follows immediately.

Corollary 2. Under the assumptions of Corollary 1, then

$$
\lim _{n} \sigma_{n}^{-1}\left[\tau_{n}\left(t_{i}, t_{j} ; \omega\right)-\sum_{k=1}^{n} w_{k} \delta_{t_{i}}\left(\xi_{k-1}\right) p_{k}\left(t_{i}, t_{j}\right)\right]=0 \quad a . s .
$$

Proof: Let $g(x, y)=\delta_{t_{i}}(x) \delta_{t_{j}}(y), x, y \in \mathcal{E}$ in Theorem 2, we have

$$
\begin{aligned}
& \sum_{k=1}^{n} w_{k}\left[f\left(\xi_{k-1}, \xi_{k}\right)-\sum_{t_{l} \in \mathcal{E}} g\left(\xi_{k-1}, t_{l}\right) p_{k}\left(\xi_{k-1}, t_{l}\right)\right] \\
= & \sum_{k=1}^{n} w_{k}\left[\delta_{i}\left(\xi_{k-1}\right) \delta_{j}\left(\xi_{k}\right)-\sum_{t_{l} \in \mathcal{E}} \delta_{t_{i}}\left(\xi_{k-1}\right) \delta_{t_{j}}\left(t_{l}\right) p_{k}\left(\xi_{k-1}, t_{l}\right)\right] \\
= & \tau_{n}\left(t_{i}, t_{j} ; \omega\right)-\sum_{k=1}^{n} w_{k} \delta_{t_{i}}\left(\xi_{k-1}\right) p_{k}\left(t_{i}, t_{j}\right)
\end{aligned}
$$


Theorem 3. Let $\left\{\xi_{n}, n \geq 0\right\}$ be an inhomogeneous Markov chain defined by (1.1),(1.2), $\tau_{n}\left(t_{i} ; \omega\right), \tau_{n}\left(t_{i}, t_{j} ; \omega\right),\left\{\sigma_{n}, n \geq 1\right\}$ be defined as before with $\sigma_{n} \uparrow$ $\infty$ a.s., Let $P$ be another transition matrix and irreducible. If

$$
\lim _{n} \sigma_{n}^{-1} \sum_{k=1}^{n}\left|w_{k} p_{k}\left(t_{i}, t_{j}\right)-w_{k-1} p\left(t_{i}, t_{j}\right)\right|=0 \text { for all } t_{i}, t_{j} \in \mathcal{E}
$$

then

(1) $\lim _{n} \frac{\tau_{n}\left(t_{i} ; \omega\right)}{\sigma_{n}}=\pi_{i} ;$ a.s.

(2) $\lim _{n} \frac{\tau_{n}\left(t_{i}, t_{j} ; \omega\right)}{\sigma_{n}}=\pi_{i} p\left(t_{i}, t_{j}\right) ; \quad$ a.s.

(3) $\lim _{n} \frac{1}{\sigma_{n}} \sum_{k=1}^{n} w_{k} g\left(\xi_{k-1}, \xi_{k}\right)=\sum_{t_{i} \in \mathcal{E}} \pi_{i} \sum_{t_{j} \in \mathcal{E}} g\left(t_{i}, t_{j}\right) p\left(t_{i}, t_{j}\right)$. a.s.

where $\left(\pi_{1}, \cdots, \pi_{m}\right)$ is the unique stationary distribution determined by the transition matrix $P$.

Proof (1): From Corollary 1 we have

$$
\lim _{n} \sigma_{n}^{-1}\left|\tau_{n}\left(t_{j} ; \omega\right)-\sum_{k=1}^{n} w_{k} p_{k}\left(\xi_{k-1}, t_{j}\right)\right|=0
$$

Since

$$
\sum_{k=1}^{n} w_{k} p_{k}\left(\xi_{k-1}, t_{j}\right)=\sum_{k=1}^{n} \sum_{t_{i} \in \mathcal{E}} w_{k} \delta_{t_{i}}\left(\xi_{k-1}\right) p_{k}\left(t_{i}, t_{j}\right)
$$

Hence

$$
\begin{aligned}
& \lim _{n} \sigma_{n}^{-1}\left|\sum_{k=1}^{n} \sum_{t_{i} \in \mathcal{E}}\left[w_{k} \delta_{i}\left(\xi_{k-1}\right) p_{k}\left(t_{i}, t_{j}\right)-w_{k-1} \delta_{i}\left(\xi_{k-1}\right) p\left(t_{i}, t_{j}\right)\right]\right| \\
\leq & \sum_{t_{i} \in \mathcal{E}} \lim _{n} \sigma_{n}^{-1} \sum_{k=1}^{n}\left|w_{k} p_{k}\left(t_{i}, t_{j}\right)-w_{k-1} p\left(t_{i}, t_{j}\right)\right|=0
\end{aligned}
$$

Noticing that $\sum_{t_{i} \in \mathcal{E}} \frac{\tau_{n}\left(t_{i} ; \omega\right)}{\sigma_{n}}=1$, we have

$$
\begin{aligned}
& \lim _{n} \sigma_{n}^{-1}\left[\tau_{n}\left(t_{j} ; \omega\right)-\sum_{t_{i} \in \mathcal{E}} \tau_{n}\left(t_{i} ; \omega\right) p\left(t_{i}, t_{j}\right)\right] \\
& \lim _{n} \sigma_{n}^{-1} \mid \sum_{k=1}^{n} \sum_{t_{i} \in \mathcal{E}}\left\{\left[w_{k} \delta_{t_{i}}\left(\xi_{k-1}\right) p_{k}\left(t_{i}, t_{j}\right)-w_{k-1} \delta_{i}\left(\xi_{k-1}\right) p\left(t_{i}, t_{j}\right)\right]\right. \\
& \left.\quad+\left[w_{0} \delta_{i}\left(\xi_{0}\right) p\left(t_{i}, t_{j}\right)-w_{n} \delta_{i}\left(\xi_{n}\right) p\left(t_{i}, t_{j}\right)\right]\right\} \mid, \quad t_{j} \in \mathcal{E}
\end{aligned}
$$


Multiplying (3.9) by $p\left(t_{j}, t_{k}\right)$, adding them together for $t_{j} \in \mathcal{E}$, and using (3.9) once again, we have

$$
\begin{aligned}
0 & =\sum_{t_{j} \in \mathcal{E}} p\left(t_{j}, t_{k}\right) \lim _{n} \sigma_{n}^{-1}\left[\tau_{n}\left(t_{j} ; \omega\right)-\sum_{t_{i} \in \mathcal{E}} \tau_{n}\left(t_{i} ; \omega\right) p\left(t_{i}, t_{j}\right)\right] \\
& =\lim _{n}\left[\sum_{t_{j} \in \mathcal{E}} \frac{\tau_{n}\left(t_{i} ; \omega\right)}{\sigma_{n}} p\left(t_{j}, t_{k}\right)-\frac{\tau_{n}\left(t_{k} ; \omega\right)}{\sigma_{n}}\right] \\
& +\lim _{n}\left[\frac{\tau_{n}\left(t_{k} ; \omega\right)}{\sigma_{n}}-\sum_{t_{j} \in \mathcal{E}} \sum_{t_{i} \in \mathcal{E}} \frac{\tau_{n}\left(t_{i} ; \omega\right)}{\sigma_{n}} p\left(t_{i}, t_{j}\right) p\left(t_{j}, t_{k}\right)\right] \\
& =\lim _{n}\left[\frac{\tau_{n}\left(t_{k} ; \omega\right)}{\sigma_{n}}-\sum_{t_{i} \in \mathcal{E}} \frac{\tau_{n}\left(t_{i} ; \omega\right)}{\sigma_{n}} p^{(2)}\left(t_{i}, t_{k}\right)\right]
\end{aligned}
$$

where $p^{(l)}\left(t_{i}, t_{k}\right)$ is the $l$-step transition of $\mathrm{P}$. We show by induction that

$$
\lim _{n}\left[\frac{\tau_{n}\left(t_{k} ; \omega\right)}{\sigma_{n}}-\sum_{t_{i} \in \mathcal{E}} \frac{\tau_{n}\left(t_{i} ; \omega\right)}{\sigma_{n}} p^{(l)}\left(t_{i}, t_{k}\right)\right]=0 \quad \text { a.s. }
$$

We conclude that

$$
\lim _{n}\left[\frac{\tau_{n}\left(t_{k} ; \omega\right)}{\sigma_{n}}-\sum_{t_{i} \in \mathcal{E}} \frac{\tau_{n}\left(t_{i} ; \omega\right)}{\sigma_{n}} \frac{1}{h} \sum_{l=1}^{h} p^{(l)}\left(t_{i}, t_{k}\right)\right]=0 \text { a.s. }
$$

Since

$$
\lim _{h}\left[\frac{1}{h} \sum_{l=1}^{h} p^{(l)}\left(t_{i}, t_{k}\right)\right]=\pi_{k} \text { for all } t_{i} \in \mathcal{E}
$$

These together with $\sum_{t_{i} \in \mathcal{E}}^{m} \frac{\tau_{n}\left(t_{i} ; \omega\right)}{\sigma_{n}}=1$ yield the recursion formulas (3.6).

(2):By Corollary 2, we have

$$
\lim _{n} \sigma_{n}^{-1}\left[\tau_{n}\left(t_{i}, t_{j} ; \omega\right)-\sum_{k=1}^{n} w_{k} \delta_{t_{i}}\left(\xi_{k-1}\right) p_{k}\left(t_{i}, t_{j}\right)\right]=0
$$

It is easy to see from (3.14) that

$$
\lim _{n} \sigma_{n}^{-1} \sum_{k=1}^{n} \delta_{i}\left(\xi_{k-1}\right)\left[w_{k} p_{k}\left(t_{i}, t_{j}\right)-w_{k-1} p\left(t_{i}, t_{j}\right)\right]=0
$$

These imply

$$
\begin{aligned}
& \lim _{n}\left[\frac{\tau_{n}\left(t_{i}, t_{j} ; \omega\right)}{\sigma_{n}}-\frac{\tau_{n}(i ; \omega)}{\sigma_{n}} p\left(t_{i}, t_{j}\right)\right]=\lim _{n} \sigma_{n}^{-1} \sum_{k=1}^{n} \delta_{t_{i}}\left(\xi_{k-1}\right)\left\{\left[w_{k} p_{k}\left(t_{i}, t_{j}\right)\right.\right. \\
& \left.\left.-w_{k-1} p\left(t_{i}, t_{j}\right)\right]-\left[w_{n} \delta_{t_{i}}\left(\xi_{n}\right)+w_{0} \delta_{t_{i}}\left(\xi_{0}\right)\right] p\left(t_{i}, t_{j}\right)\right\}=0
\end{aligned}
$$


(3.7) follows.

(3):Since

$$
\begin{aligned}
\frac{1}{\sigma_{n}} \sum_{k=1}^{n} w_{k} g\left(\xi_{k-1}, \xi_{k}\right) & =\frac{1}{\sigma_{n}} \sum_{k=1}^{n} \sum_{t_{i} \in \mathcal{E}} \sum_{t_{j} \in \mathcal{E}} w_{k} \delta_{i}\left(\xi_{k-1}\right) \delta_{t_{j}}\left(\xi_{k}\right) g\left(t_{i}, t_{j}\right) \\
& =\sum_{t_{i} \in \mathcal{E}} \sum_{t_{j} \in \mathcal{E}} g\left(t_{i}, t_{j}\right) \frac{\tau_{n}\left(t_{i}, t_{j} ; \omega\right)}{\sigma_{n}}
\end{aligned}
$$

thus (3.8) follows from (3.6) and (3.7).

Corollary 3. (See e.g. [6])Let $\left\{\xi_{n}, n \geq 0\right\}, P$ be defined as before. If

$$
\lim _{n} n^{-1} \sum_{k=1}^{n}\left|p_{k}\left(t_{i}, t_{j}\right)-p\left(t_{i}, t_{j}\right)\right|=0 \text { for all } t_{i}, t_{j} \in \mathcal{E}
$$

then

(1) $\lim _{n} \frac{\kappa_{n}\left(t_{i} ; \omega\right)}{n}=\pi_{i} ; \quad$ a.s.

(2) $\lim _{n} \frac{\kappa_{n}\left(t_{i}, t_{j} ; \omega\right)}{n}=\pi_{i} p\left(t_{i}, t_{j}\right) ; \quad$ a.s.

(3) $\lim _{n} \frac{1}{n} \sum_{k=1}^{n} g\left(\xi_{k-1}, \xi_{k}\right)=\sum_{t_{i} \in \mathcal{E}} \pi_{i} \sum_{t_{j} \in \mathcal{E}} g\left(t_{i}, t_{j}\right) p\left(t_{i}, t_{j}\right)$. a.s.

where $\kappa_{n}\left(t_{j} ; \omega\right)=\sum_{k=1}^{n} \delta_{t_{j}}\left(\xi_{k}\right), \kappa_{n}\left(t_{i}, t_{j} ; \omega\right)=\sum_{k=1}^{n} \delta_{t_{i}}\left(\xi_{k-1}\right) \delta_{t_{j}}\left(\xi_{k}\right)$.

Proof: Putting $w_{n} \equiv 1$ in Theorem 3, it follows.

\section{REFERENCES}

[1] D.L. Burkholder, Martingale transforms, Ann. Math. Statist. 37 (1966), 1494-1504.

[2] W.F. Stout, All most sure convergence, Academic Press, New York, 1974.

[3] R.V. Mises, Mathematical Theory of Probability and Statistics, Academic Press, New York, 1964.

[4] W. Liu, Z.Z. Wang, An extension of a theorem on gambling systems to arbitrary binary random variables, 28( 1996 ), 51-58.

[5] A.N. Kolmogorov, On the logical foundations of probability theory, Lecture Notes in Mathematics, Springer-Verlag, New York, (1982),Vol.1021,1-5.

[6] W.G. Yang, W. Liu, The asymptotic equipartition property for $\mathrm{m}$ order nonhomogeneous Markov information source, Acta Mathematicae Applicatae Sinica, 45(4)( 2002), 686-693.

Received: June 10, 2007 